\title{
PHENOMENAL REPORTS \\ Enhanced motion during gradual changes in luminance polarity
}

\author{
Stuart Anstis* (1) \\ Department of Psychology, UC San Diego, La Jolla, CA, USA
}

Abstract

A pattern that gradually reverses its polarity as it moves can appear to move farther and faster than it really does. I shall call this new effect 'enhanced motion', since it accentuates the amplitude and briskness of motion as opposed to reverse phi, in which polarity changes reverse the perceived direction of a moving object.

Keywords: motion; reverse-phi; peripheral vision

To access the movies for this article, please visit the article landing page or read the html version of the article where all movies are embedded.

\author{
Edited by: \\ Takahiro Kawabe \\ NTT Communication Science \\ Laboratories, Japan

\section{Reviewed by:} \\ George Mather \\ University of Lincoln, UK \\ Arthur Shapiro \\ American University, USA
}

I report a novel illusion of seen movement: a pattern that gradually reverses its luminance polarity as it moves can appear to move farther and faster than it really does. I call this effect 'enhanced movement'. Thus, a black square that moves through a small distance (say $12 \mathrm{~min}$ arc) in $1 \mathrm{~s}$ and smoothly changes to white as it moves will appear to move up to twice as far as it really does.

To put this in context, I begin with an informal review of my own work over the years on luminance and motion.

\section{Luminance and motion}

Visual motion is nothing more than changes in luminance over space and time. A static spot conveys almost no information. A flickering spot changes its luminance over time but not space, and it can pump out a Morse code of information at a rate that is limited only by its highest frequency. A spatial array of dots can be arranged to vary in luminance (black or white) over space but not time, and such an array can convey a huge amount of information. A set of such dots spatially arranged to convey information is a book. Finally, dots or pixels that vary in both space and time constitute motion, and these convey the highest rate of information of all. Perhaps that is why television is more popular than books, especially among the young. Technology has followed a similar path. Writing is one of the most ancient arts. Recorded sound - a single waveform varying over time - was first captured by Edison's phonograph in 1877. To accompany the phonograph, Edison commissioned his laboratory assistant Dickson to invent a motion-picture camera in 1888 - a device that would 'do for the eye what the phonograph does for the ear' - record and reproduce objects in motion.

This was a major technical advance. But the time was not yet ripe for experimental manipulations of motion. Rotating disks viewed through a slit can only take you so far. Computers, once they arrived, gave us the means to manipulate motion. So when the main instruments available to psychologists were initially pen and ink, the illusions they usually studied were stationary geometrical patterns. Only with computers could they start studying motion perception in earnest. Perhaps that is why 'motion illusions' are mentioned on Google twice as often as 'geometrical illusions' and three or four times more often than face, colour or brightness illusions (Shapiro \& Todorovich, 2017).

\section{Luminance: Changes over time but not space}

My first faltering steps to study changes in luminance began when I was a graduate student at Cambridge under Richard Gregory. I discovered the ramp after-effect quite by chance while I was looking for something else. The eminent Scottish physicist Donald MacKay had recently discovered a motion illusion (1958) in which he waved a small illuminated bulb around under a flashing stroboscope. The continuously visible glowing filament seemed to separate out from the intermittently lit glass shell of the bulb, and 
I wanted to find out why. I wondered whether it was the onset or the offset of the flash that was more important. These days it would be very easy to do this on the computer display, but this was many years before I ever saw a computer. So, to make my sawtooth lamp, I took a large fluorescent tube and built a power pack to drive it. In parallel with the lamp, I put a large rotating potentiometer that I could rotate with a geared-down electric motor. This took me at least a couple of weeks to build. When all was built, I switched on the power and the motor moved noisily round - and the brightness of the tube gradually increased to a maximum and then abruptly fell back to zero, repetitively, as many times as I wanted, just as I had intended. Then, I turned off the power to switch the light to a steady level. To my surprise, the lamp appeared to be dimming steadily for several seconds. I was horrified that there was something wrong with my power pack, and I spent the next week disassembling it and trying to repair it. It was only after a week that it occurred to me that I had stumbled across a new visual after-effect. If you adapt to a repetitively sawtoothing light, a steady light will appear to be gradually dimming and vice versa. I found that if the test field was a spatial gradient, changing from dark on the left to light on the right, a dimming after-effect gave an impression of movement to the right. My conclusion was that the visual system contains units tuned to gradual change of luminance. These could be like a standard Reichardt (1961) unit, the difference being that Reichardt units have two inputs in different places, whereas these brightening or dimming detectors would have the two units in the same place. These might be analogues of the dimming detectors first found in the frog by Lettvin, Maturana, McCulloch and Pitts (1961).

I published this article in Science (1967). In fact, this was the only article I ever published in Science.

\section{Luminance and motion: Complex patterns}

I next turned my attention to visual motion. This was at a time when computers began to be useful in vision research. I never saw a computer myself but I was fascinated with the new work by Bela Julesz who studied stereo vision using random dot patterns, culminating in his wonderful 1970 book, which is still worth reading today. So I photocopied some of his random patterns and pasted them around a kymograph drum. I lit them with a strobe and varied the flash rate until one could just see the direction in which the random dots were moving. Results were promising but never quite clear. In other words, I almost discovered Dmax. Almost but not quite. I failed, where later on Baker and Braddick (1985) succeeded. They succeeded in finding Dmax where I failed. But I did it first.

\section{Luminance and motion: Reverse phi}

I realised that I had bitten off more than I could chew, so I retrenched and simplified my stimuli. I still used random dot patterns stolen from Julesz, but instead of a long sequence of flashes I now presented only two identical patterns in alternation. Again, this was before the days of PowerPoint, so I asked my photographer to make slides out of Julesz' random patterns. A slide is simply a piece of transparent film mounted on a cardboard holder and enlarged onto a screen with a projector. I projected two of these overlapping on a screen, and I could cut or dissolve between them by putting my hand over the lenses either rapidly or slowly.

First I went to collect my new slides from my photographer, and I saw a roll of film lying on his desk. What's that? I asked. Those are the negatives of your slides he replied, and I'm about to toss them out. Don't do that please I said, cheap as muck, but please mount them as slides in little cardboard holders. So now I had twice as many slides for the same money.

I put one slide into each projector and alternated them by covering and uncovering the projectors with my hands. Rapid hand movements gave a cut, slow ones gave a dissolve. To my surprise, the movement seemed to go in the wrong direction - backwards instead of forwards. When I examined the slides, I found that I had accidentally put a positive slide in one projector and a negative in the other one. This made the polarities reverse between successive frames, and the result was that the perceived motion went in the reverse direction towards the earlier stimulus. I had stumbled across 'reverse phi'. Reverse-phi stimuli have subsequently been used to investigate neural mechanisms of visual processing in a wide variety of animals such as monkeys, cats, wallabies, beetles, fruit flies and zebrafish (review: Kirkels, Zhang, Duijenhouwer, \& van Wezel, 2020).

Later on, I elaborated this into a four-stroke repetitive movie containing only four frames, two positive and two negative. Since then Shioiri and Cavanagh (1990) reduced this to a three-stroke cycle, and Mather and Challinor (2009) simplified it further into a two-stroke cycle, in both cases by interpolating negative after-images into the sequence. Various motion illusions are demonstrated on these websites:

\section{http://www.georgemather.com/MotionMP4.html https://cavlab.net/Demos/ http://anstislab.ucsd.edu/illusions/}

\section{Two spots}

These moving random-dot patterns were too complex for me to understand fully, so I simplified matters even further by looking at only one or two moving spots. One of the simplest possible motion stimuli is a black spot on a grey surround that suddenly disappears and simultaneously reappears nearby. In theory, we might see this as two unrelated events, but the visual system is extremely good 
at spotting such 'suspicious coincidences' (Barlow, 1985), and in practice, we nearly always see apparent movement of a black spot from one position to another. I added a white spot just to the right of the black spot. I made the two spots suddenly exchange luminances, so now I had a white spot on the left and a black spot on the right. Nothing actually moves, only the luminances change. What do we see? Do we see a white spot jumping to the left or a black spot jumping to the right? It all depends upon the surround. On a light surround, you see the black spot jumping left, and on the dark surround, you see the white spot jumping right. So when two opposed motions are in conflict, it is the motion with the higher contrast that wins. Next, I embedded each spot in a long vertical strip, as in White's effect. It turns out that the embedding strips have a far greater effect upon the direction of the motion than does the remainder of the surround. With light strips, even on a dark surround, the black spot will be seen as jumping, and conversely with dark strips, even on a light surround, the white spot appears to move. The moral of this story was that luminance of itself was not important. It was the motion with the higher contrast (Morgan \& Chubb, 1999) that won the day.

\section{Spots plus stripes:The footsteps illusion}

A chance visit to an art gallery led me to go back to my two moving spots, but now I added a striped background. I was looking at an exhibition of op-art paintings by the British artist Bridget Riley. I walked around a corner and came upon one of her dazzling patterns of repetitive vertical stripes, which we would now call a grating. My parallax view of the vertical edge of the corner wall as I moved happened to scan across the painting, and I noticed that this edge seemed to move jerkily instead of smoothly as it occluded or uncovered the painting. To find out what was happening, I stood still, shut one eye and moved my head from side to side, to the surprise of the gallery attendant. Back in the lab, I moved two spots smoothly along parallel paths across a surround of stationary vertical stripes. One spot was light yellow, the other was dark blue.

\section{Result}

When the blue spot lay on white stripes, it appeared to speed up, and when it lay on black stripes, it appeared to slow down. The opposite was true for the yellow spots. This confirmed the importance of luminance contrast, which here drove the apparent speed. It also drove motion salience in the two-spot competition described earlier. Full details can be found in the review of the footsteps illusion by Kitaoka \& Anstis (2021) (he has charitably included me as a co-author).

I now describe 'enhanced motion', a new example of luminance contrast driving motion salience. As with many

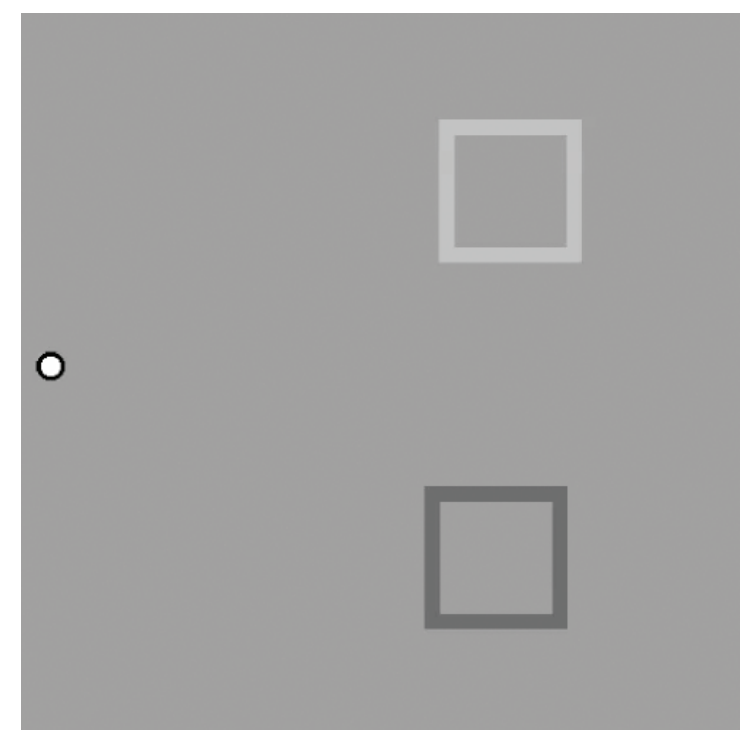

Movie 1. Enhanced versus real movement, as measured in Experiment 1.

other phenomena that I have discovered, I do not fully understand this effect. At least I am consistent.

Movie 1 shows enhanced movement. Two outline squares move back and forth continually at $1 \mathrm{~Hz}$ through the same amplitude of one line-width. The lower square is always black, but the upper square changes its luminance smoothly as it moves, being black at the left-hand end of its motion path and white at the right-hand end. Pay attention to the apparent amplitude or path length of this square. It appears to move through a greater distance than the lower square (which moves in counterphase to avoid any perceptual locking). The enhancement is strongest in peripheral vision.

We measured this illusory effect by showing observers a display similar to Movie 1 . Each square subtended a visual angle of $2^{\circ}$, and its lines were $0.2^{\circ}$ thick. The upper, polarity-reversing square was randomly set on each trial to move back and forth through $6,12,18$ or $24 \mathrm{~min}$ arc $(0.5,1,1.5$ or 2 line-widths $)$, and the observer struck designated keys to adjust the amplitude of the lower black square to an apparent match with the upper square. The squares were viewed at an eccentricity of $8^{\circ}$.

Results are graphed in Fig. 1. Figure 1a shows the raw matches made, with $x=$ actual movement of the polarity-reversing test square and $y=$ matching amplitudes selected. The judged amplitudes increased with actual amplitude, and all polarity-reversing movements were overestimated (all datum points lie above the unit-slope line). This overestimation showed diminishing returns: Fig. $1 \mathrm{~b}$ replots the same data to show the ratio between actual and perceived motion. In this plot, a reading of 

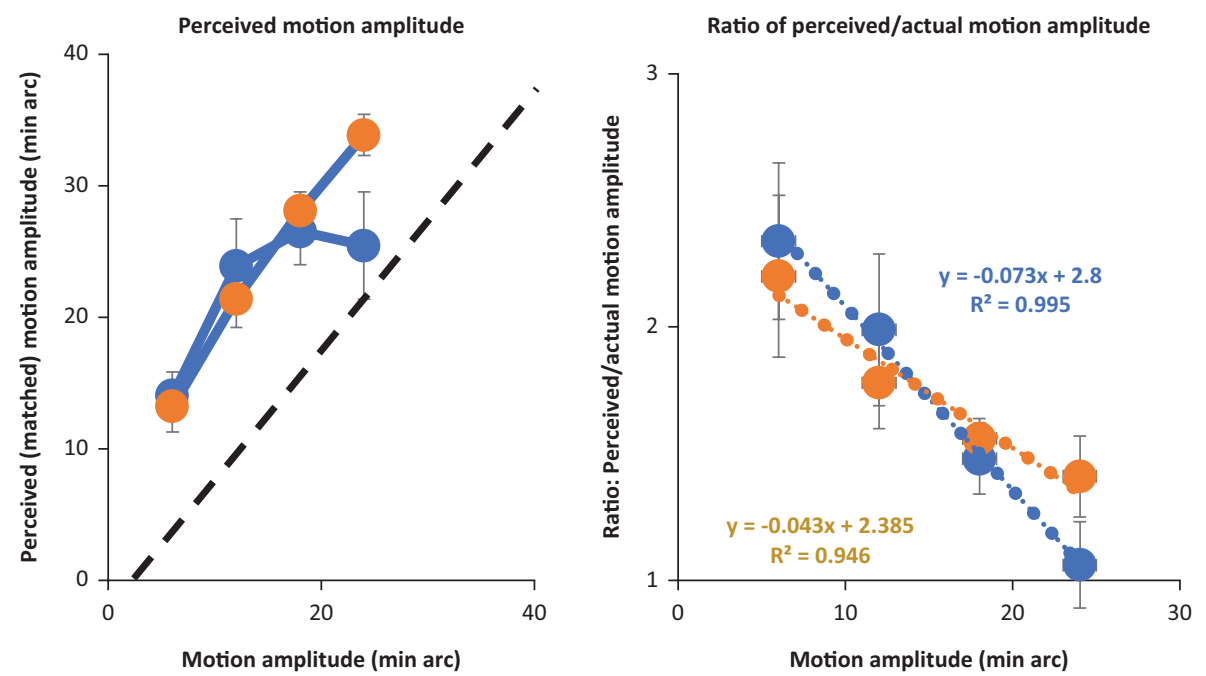

Fig. 1. (a) Perceived versus actual motion during polarity reversal (mean \pm 1 SEM for 2 observers). (b) The same data was replotted to show the ratio between actual and perceived enhanced motion. Y-values above unity indicate overestimations.

$y=1$ would mean that the perception was veridical, while $y=2$ would mean that the observer perceived the polarity-reversing motion as double its actual extent. Figure $1 \mathrm{~b}$ shows that observers strongly overestimated the condition with the smallest displacement, when the square actually moved through 6 min arc; the square appeared to move through more than twice its actual distance. Overestimation decreased with increasing actual motion, until the perception was almost veridical when the upper square moved through 24 min arc. Observers felt low confidence about the ratings that they were making in peripheral vision, but the $r^{2}>0.99$ in Fig. 1b shows that their judgements were more lawful than they thought.

Rotation gives similar effects in Movie 2, where the two wheels rotate continuously at a rate of $24 \%$ (4 rev per min), but the right-hand wheel, whose luminance changes smoothly between black and white as it moves, appears to most observers to rotate appreciably more than the all-black wheel on the left. The same is true for the expanding/contracting wheels in Movie 3. These illusory speed-ups are much more marked in peripheral vision.

What matters is not the raw luminance of the moving square but its luminance polarity against its surround. In Movie 4, the luminance of the upper square always swings between approximately 40 and 60\% (0 = 'black', $100=$ 'white'). It shows an illusory speed-up only when the surround luminance is at a mid-grey of approximately $50 \%$ (b) and not when the surround is either black (a) or white (c). So, the square needs to switch its polarity by being alternately lighter and darker than the surround luminance.

\section{Reverse phi}

(Anstis, 1970; Anstis \& Rogers, 1975; Bours, Kroes \& Lankheet, 2009; Kitaoka, 2006; Rogers, Anstis, Ashida, \& Kitaoka, 2019) also involves moving patterns that change their polarity, but it is quite different from enhanced movement. In reverse phi, the square appears to move opposite to its true direction, whereas in enhanced motion its direction is seen correctly but its amplitude is overestimated.

Movie 5 has four columns, $\mathbf{a}, \mathbf{b}, \mathbf{c}, \mathbf{d}$.

a. Control condition: a simple black square that moves left and right.

b. Same square as a but reversing in polarity, changing smoothly from black to white as it moves. This is the enhanced motion that is the topic of this article. b would be seen as moving in the same direction as a, but with an illusory enhanced amplitude.

c. 'Cutting' version of reverse phi, which is the same as a except that it reverses the polarity on every frame.

d. 'Dissolving' version of reverse phi. A dark square gradually fades down, while a light square, displaced to the right, gradually fades up.

Perceived motion for $\mathbf{c}$ and $\mathbf{d}$ would be backwards.

Movie 5 shows cartoon versions of enhanced notion (b) and reverse-phi motion (c and d). Column a shows a black control square moving back and forth. b shows enhanced movement, while c and d show 'cutting' and 'dissolving' versions of reverse phi (Rogers et al., 2019). Reverse-phi is strongest in peripheral vision (Edwards \& Nishida, 2004; Lorenceau \& Cavanagh, 2020) and appears to move backwards, so squares $\mathbf{c}$ and $\mathbf{d}$ would move 


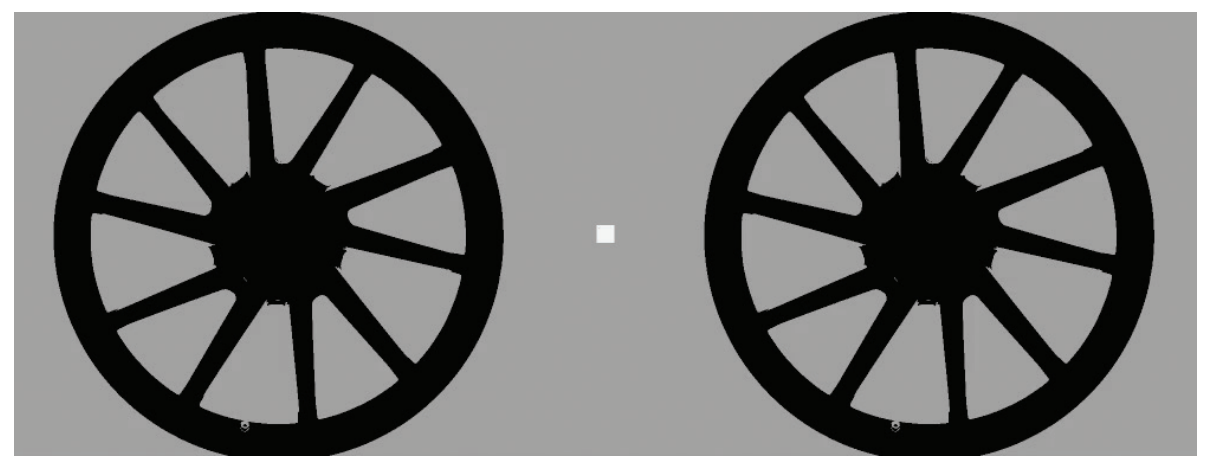

Movie 2. Wheel on the right looks faster.

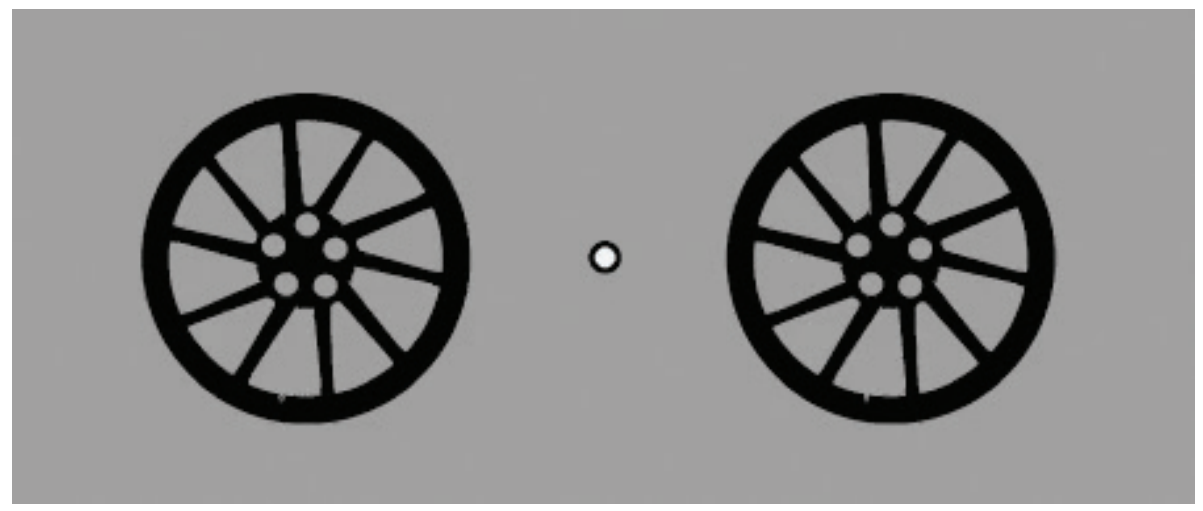

Movie 3. Wheel on the right seems to expand and contract more.

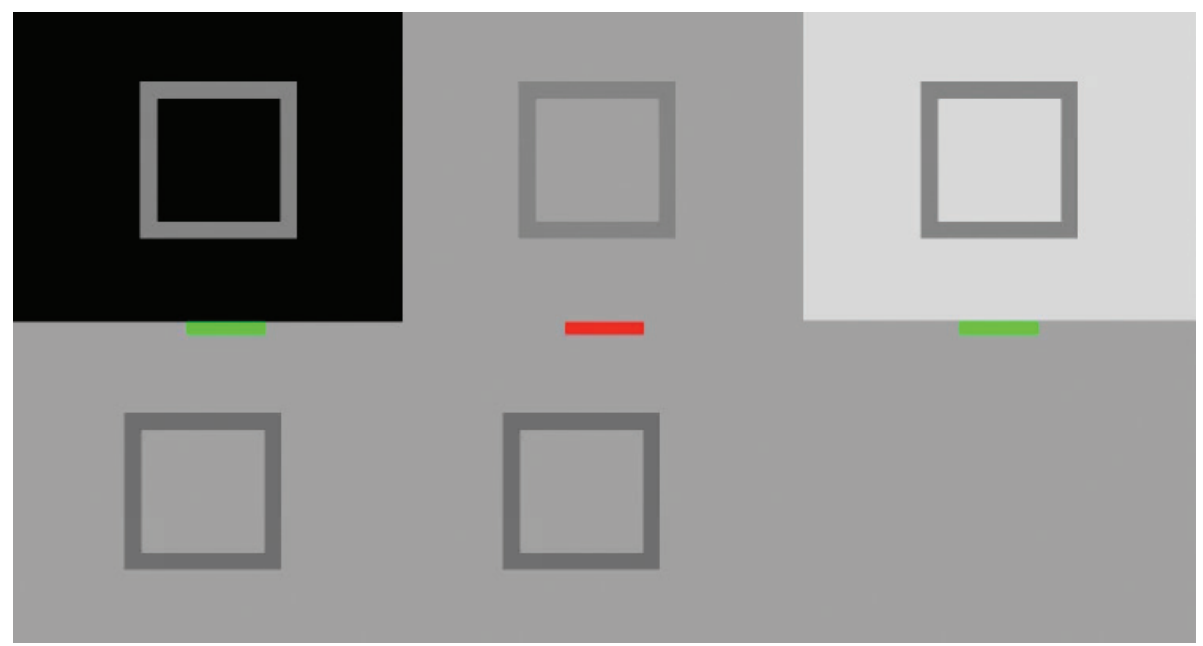

Movie 4. Upper square shows enhanced movement, not in (a) or (c) but only in (b), where the luminance of the grey surround is straddled by the light and dark levels of the upper square. Try covering the unattended stimuli with your hands.

in the opposite direction from $\mathbf{a}$ and $\mathbf{b}$. Movie 5 demonstrates that enhanced movement and reverse-phi are quite different phenomena.

We do not yet know what causes this apparent speed-up, but we know some things that do not. Reverse phi involves moving objects that change their polarity, but the present effect enhances motion while reverse phi reverses its direction, so the two effects are quite different. We wondered whether randomly flickering the moving square might activate motion detectors in some non-specific way to make the display 


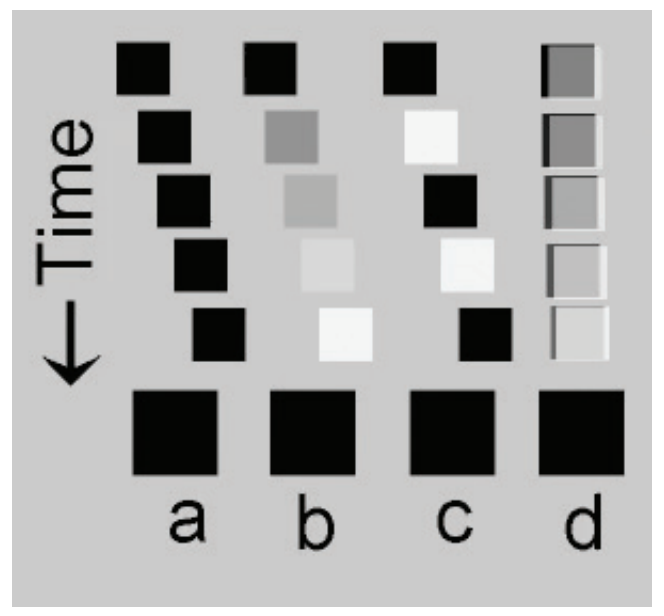

Movie 5. Cartoons of stimuli that produce enhanced and reversed movement. Time runs down the page. Rows 1-5 in each column show a sequence of five sample frames as a square moves to the right (and back again, not shown). The bottom row animates these sequences in slow motion. These are merely diagrams not intended to show the perceptual speed-ups or reversals.

look more dynamic, but we have found that the enhanced motion disappears if a moving square simply flickers randomly. Polarity is important - in Movie 4, the mid-grey surround makes the upper square change its polarity, and mere swings in luminance are not enough, since the effect disappears for a black or white surround that lies outside the range of the square's varying luminance.

We speculate that enhanced motion occurs because increasing the luminance contrast of a moving object increases the salience of its motion (Anstis, 2001; Morgan \& Chubb, 1999; Thompson, 1982). Continuous brief adaptation to the modulating square (successive contrast) makes it look alternately whiter than white and blacker than black as it moves, and that this subjective increase in Michelson contrast might enhance the salience of the motion.

Also, a moving square may first stimulate neural OFF channels (Schiller, 1992) when it is initially black and then stimulate ON channels when it becomes white. This splitting of the motion between separate neural pathways might possibly make the two halves of motion look further apart.

But we have not yet determined the crucial differences in stimulus properties that cause enhanced motion and reverse-phi to produce opposite visual effects.

\section{Acknowledgements}

Thanks to Neal Dykmans, Sae Kaneko, and Don Macleod, and the referees George Mather and Art Shapiro for their helpful comments and discussions.

\section{Conflict of interest and funding}

The author declares no conflicts of interest. This study was supported by a grant from the UCSD Dept of Psychology.

\section{References}

Anstis, S. (2001). Footsteps and inchworms: Illusions show that contrast affects apparent speed. Perception, 30(7), 785-794. doi: $10.1068 / \mathrm{p} 3211$

Anstis, S. M. (1967). Visual adaptation to gradual change of intensity. Science, 155(3763), 710-712. doi: 10.1126/science. 155.3763 .710

Anstis, S. M. (1970). Phi movement as a subtraction process. Vision Research, 10, 1411-1430. doi: 10.1016/ 0042-6989(70)90092-1

Anstis, S. M., \& Rogers, B. J. (1975). Illusory reversal of visual depth and movement during changes of contrast. Vision Research, 15, 957-961. doi: 10.1016/0042-6989(75)90236-9

Baker, C. L., \& Braddick, O. J. (1985). Eccentricity-dependent scaling of the limits for short-range apparent motion perception. Vision Research, 25(6), 803-812. doi: 10.1016/00426989(85)90188-9

Barlow, H. B. (1985). Cerebral cortex as a model builder. In V. D. D. Rose (Ed.), Models of the visual cortex (p. 37C46). New York: John Wiley.

Bours, R. J., Kroes, M. C., \& Lankheet, M. J. (2009). Sensitivity for reverse-phi motion. Vision Research, 49(1), 1-9. doi: 10.1016/j. visres.2008.09.014

Edwards, M., \& Nishida, S. (2004). Contrast-reversing globalmotion stimuli reveal local interactions between first- and second-order motion signals. Vision Research, 44(16), 1941-1950. doi: 10.1016/j.visres.2004.03.016

Julesz, B. (1970). Foundations of cyclopean perception. Chicago: University of Chicago Press.

Kirkels, L. A. M. H., Zhang, W., Duijenhouwer, J., \& van Wezel, R. J. A. (2020). Opto-locomotor reflexes of mice to reverse-phi stimuli. Journal of Vision, 20, 7. doi: 10.1167/jov.20.2.7

Kitaoka, A. (2006). Configurational coincidence among six different phenomena: A comment on van Lier and Csathó. Perception, 35, 799-806. doi: 10.1068/p5319b

Kitaoka, A., \& Anstis, S. (2021). A review of the footsteps illusion. Journal of Illusion, 2(1), 5612. doi: 10.47691/joi. v2.5612

Lettvin, J. Y., Maturana, H. R., McCulloch, W. S., \& Pitts, W. H. (1961). Two remarks on the visual system of the frog. In W. A. Rosenblith (Ed.), Sensory communication (pp. 757-776). Cambridge, MA: MIT Press. Retrieved from https://cepa. info/524

Lorenceau, J., \& Cavanagh, P. (2020). Jumpy and jerky: When peripheral vision faces reverse-phi. i-Perception, 11(5), 1-5. doi: $10.1177 / 2041669520939107$

Mackay, D. M. (1958). Perceptual stability of a stroboscopically lit visual field containing self-luminous objects. Nature, 181(4607), 507-8. doi: 10.1038/181507a0

Mather, G., \& Challinor, K. L. (2009). Psychophysical properties of two-stroke apparent motion. Journal of Vision, 9(1), 28, 1-6. doi: $10.1167 / 9.1 .28$

Morgan, M. J., \& Chubb, C. (1999). Contrast facilitation in motion detection: Evidence for a Reichardt detector in human vision. Vision Research, 39(25), 4217-4231. doi: 10.1016/ s0042-6989(99)00136-4

Reichardt, W. (1961). Autocorrelation: A principle for the evaluation of sensory evidence by the central nervous system. In W. Rosenblith (Ed.), Sensory communication (pp. 303-318). Cambridge, MA: MIT Press.

Rogers, B. J., Anstis, S., Ashida, H., \& Kitaoka, A. (2019). Reversed phi and the 'Phenomenal Phenomena' revisited. I-Perception, 10, 4. doi: $10.1177 / 2041669519856906$ 
Schiller, P. H. (1992). The ON and OFF channels of the visual system. Trends in Neurosciences, 15(3), 86-92. doi: 10.1016/ 0166-2236(92)90017-3

Shapiro, A. G., \& Todorovich, D. (2017). Introduction, Figure 2. In A. G. Shapiro \& D. Todorovich (Eds.), The Oxford compendium of visual illusions. Oxford: Oxford University Press.
Shioiri, S., \& Cavanagh, P. (1990). ISI produces reverse apparent motion. Vision Research, 30(5), 757-768. doi: 10.1016/ 0042-6989(90)90101-p

Thompson. P. (1982). Perceived rate of movement depends on contrast. Vision Research, 22, 377-380. doi: 10.1016/0042-6989(82) 90153-5 